\title{
Global existence for coupled reaction diffusion systems modelling some reversible chemical reactions
}

\author{
Said Kouachi
}

Communicated by Y. Charles Li, received October 14, 2010.

\begin{abstract}
The purpose of this paper is to prove global existence of solutions for coupled reaction diffusion equations describing some coupled reversible chemical reactions. In this case the nonlinearities present a difficulties since they change sign and so neither $u$ nor $v$ the concentrations of the two reactants in question is a priori bounded or at least bounded in some $L p$-space for $p$ large. Our techniques are based on Lyapunov functional method.
\end{abstract}

\section{Contents}

1. Introduction

2. Notations and preliminary observations 81

3. Statement and proof of the main results 84

References

\section{Introduction}

We consider the family of reaction-diffusion system

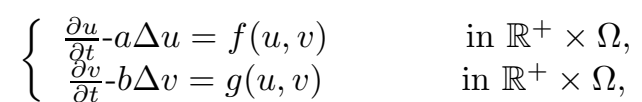

with the boundary conditions

$$
\frac{\partial u}{\partial \eta}=\frac{\partial v}{\partial \eta}=0 \quad \text { on } \mathbb{R}^{+} \times \partial \Omega
$$

and the initial data

$$
u(0, x)=u_{0}(x), \quad v(0, x)=v_{0}(x) \quad \text { in } \Omega,
$$

1991 Mathematics Subject Classification. Primary 35K45, 35K57; Secondary 35K45.

Key words and phrases. Reaction-diffusion systems, Global existence, Lyapunov functionals. This work was supported by the science college research center at Qassim university, project number SR-0-011-748. 
where

$$
f(u, v)=-h_{1} u^{l} v^{q}+h_{2} u^{r} v^{s}, g(u, v)=h_{3} u^{l} v^{q}-h_{4} u^{r} v^{s}
$$

$h_{i}, i=1,2,3$ and $4, l, q, r$ and $s$ are positive constants. $\Omega$ is an open bounded domain of class $\mathbb{C}^{1}$ in $\mathbb{R}^{n}$, with boundary $\partial \Omega, \frac{\partial}{\partial \eta}$ denotes the outward normal derivative on $\partial \Omega$ and $a$ and $b$ are positive constants. The initial data are assumed to be nonnegative.

The functions $f$ and $g$ are called the reaction terms or the nonlinearities of the system (1.1).

System (1.1) may be a model for the chemical reaction

$$
l A+q B \underset{k}{\stackrel{h}{\rightleftarrows}} r A+s B .
$$

More precisely, we have the following reaction diffusion system

$$
\begin{cases}\frac{\partial u}{\partial t}-a \Delta u=(r-l)\left[\lambda_{1} u^{l} v^{q}-\lambda_{2} u^{r} v^{s}\right] & \text { in } \mathbb{R}^{+} \times \Omega, \\ \frac{\partial v}{\partial t}-b \Delta v=(q-s)\left[-\lambda_{1} u^{l} v^{q}+\lambda_{2} u^{r} v^{s}\right] & \text { in } \mathbb{R}^{+} \times \Omega\end{cases}
$$

where $u$ and $v$ are the concentrations respectively of the reactants $A$ and $B$ and $\lambda_{1}$ and $\lambda_{2}$ are the reaction constants and $r>l$ and $q>s$ to get the same signs as in (1.1).

The difficulty for this type systems is that the reaction terms do not have a constant sign and this means that none of the equations are good in the sense that neither $u$ nor $v$ is a priori bounded or at least bounded in some $L p$-space for $p$ large in order to apply the well known regularizing effect and deduce the global existence in time for problem (1.1)-(1.3).

In the case when the nonlinearities have a constant sign many results have been obtained: When $f(u, v)=-u v^{\beta}$ (witch implies the uniform boundless of $u$ ) and $g(u, v)=u v^{\beta}$, N. Alikakos [2] established global existence and $L^{\infty}$-bounds of positive solutions when $1<\beta<\frac{(n+2)}{n}$. K. Masuda [14] showed that the solutions exist globally for every $\beta>1$. S. L. Hollis, R. H. Martin and M. Pierre [7] established global existence of positive solutions for system (1.1) with the boundary conditions

$$
\lambda_{1} u+\left(1-\lambda_{1}\right) \frac{\partial u}{\partial \eta}=\beta_{1}, \lambda_{2} v+\left(1-\lambda_{2}\right) \frac{\partial v}{\partial \eta}=\beta_{2} \quad \text { on } \mathbb{R}^{+} \times \partial \Omega
$$

where

$$
0<\lambda_{1}, \lambda_{2}<1, \lambda_{1}=\lambda_{2}=1, \beta_{1} \geq 0 \text { and } \beta_{2} \geq 0, \text { or } \lambda_{1}=\lambda_{2}=\beta_{1}=\beta_{2}=0
$$

under the conditions of the uniform boundedness of $u$ on $\left[0, T_{\max }\right] \times \Omega$ and

$$
f(r, s)+g(r, s) \leq C(r, s)(r+s+1), \text { for all } r \geq 0 \text { and } s \geq 0,
$$

with $f$ and $g$ can change sign and where $C(r, s)$ is positive and uniformly bounded function defined on $\mathbb{R}^{+} \times \mathbb{R}^{+}$. Haraux and A. Youkana [4] simplified the proof of K. Masuda while using techniques based on Lyapunov functional and while taking nonlinearities $f(u, v)=-g(u, v)=-u F(v)$ satisfying the condition

$$
\lim _{s \rightarrow+\infty}\left[\frac{\log (1+F(s))}{s}\right]=0,
$$


where $F(s) \geq 0$ for all $s \geq 0$. S. Kouachi and A. Youkana [11] generalized the results of A. Haraux and A. Youkana [4] while adding $-c \Delta u$ to the right-hand side of the second equation of system (1.1) and the condition

$$
\lim _{s \rightarrow+\infty}\left[\frac{\log (1+f(r, s))}{s}\right]<\alpha^{*}, \text { for any } r \geq 0,
$$

where $f(r, s) \geq 0$ for all $r, s \geq 0$ and

$$
\alpha^{*}=\frac{2 a b}{n(a-b)^{2}\left\|u_{0}\right\|_{\infty}}
$$

condition reflecting the weak exponential growth of the reaction term $f$. One notices that condition (1.5) is insufficient to prove global existence for solutions to system (1.1) and authors impose to $f$ (or $g$ ) to satisfy in addition the following analogous condition

$$
f(r, s)(\text { or } g(r, s)) \leq C^{\prime}(r, s)(r+s+1), \text { for all } r \geq 0 \text { and } s \geq 0 .
$$

In the case when the nonlinearities do not have a constant sign, there are not many results: A. J. Morgan [15] Generalized the results of S. L. Hollis, R. H. Martin and M. Pierre [7] to show that solutions of the m-components reaction diffusion systems exist globally $(m \geq 2)$ where also, in our case $(m=2)$, he imposed to $f$ and $f+g$ conditions (1.5) under the boundary conditions $(1.2)^{\prime}$ '. S. L. Hollis [6] extended the results, under the same conditions, to the boundary conditions (1.2)' but he took

$$
0 \leq \lambda_{1}, \lambda_{2} \leq 1, \beta_{1} \geq 0 \text { and } \beta_{2} \geq 0 .
$$

In S. Kouachi[ 13], respectively in S. Kouachi[14] and finally in S. Abdelmalek and S. Kouachi[1], we generalized the above results respectively for two, three and finally $m$ components systems:insufficient to

$$
\frac{\partial u_{i}}{\partial t}-d_{i} \Delta u_{i}=f_{i}\left(u_{1}, \ldots, u_{m}\right) \quad \text { in } \mathbb{R}^{+} \times \Omega ; i=1, \ldots, m,
$$

under the unique condition

$$
\sum_{i=1}^{m} D_{i} f_{i}\left(u_{1}, \ldots, u_{m}\right) \leq C\left[1+\sum_{i=1}^{m} u_{i}\right]
$$

for all positive constants $D_{i}$ sufficiently large, where $C$ is positive constant and we showed the global existence without imposing the boundedness of one of the components of the solution.

\section{Notations and preliminary observations}

It is well known that to prove global existence of solutions to (1.1)-(1.3) (see Henry [5]), it suffices to derive a uniform estimate of $\|f(u, v)\|_{p}$ and $\|g(u, v)\|_{p}$ on $\left[0, T^{*}[\right.$ for some $p>n / 2$. Our aim is to apply polynomial Lyapunov functional method (see M. Kirane and S. Kouachi [8], [9] and [10], S. Kouachi and A. Youkana [11] and S. Kouachi[13] and [14] and S. Abdelmalek and S. Kouachi[1]) according to the solutions $(u, v)$ of system (1.1), to carry out their $\mathrm{L}^{p}$-bounds and deduct their global existence. The nonnegativity of the solutions is preserved by application of 
classical results on invariant regions (see J. Smoller [18]), since the reaction (1.4) is quasi-positive, i.e:

$$
f(0, v) \geq 0, \quad \text { for all } v \geq 0 \text { and } g(u, 0) \geq 0, \quad \text { for all } u \geq 0 .
$$

The usual norms in the spaces $\mathbb{L}^{p}(\Omega), \mathbb{L}^{\infty}(\Omega)$ and $\mathbb{C}(\bar{\Omega})$ are respectively denoted by

$$
\begin{gathered}
\|u\|_{p}^{p}=\frac{1}{|\Omega|} \int_{\Omega}|u(x)|^{p} d x, \\
\|u\|_{\infty}=\max _{x \in \Omega}|u(x)| .
\end{gathered}
$$

Since the nonlinear right hand side of (1.1) is continuously differentiable on $\mathbb{R}^{+} \times \mathbb{R}^{+}$, then for any initial data in $\mathbb{C}(\bar{\Omega})$ or $\mathbb{L}^{p}(\Omega), p \in(1,+\infty)$, it is easy to check directly its Lipschitz continuity on bounded subsets of the domain of a fractional power of the operator

$$
\left(\begin{array}{ll}
-a \Delta & 0 \\
0 & -b \Delta
\end{array}\right)
$$

Under these assumptions, the following local existence result is well known (see A. Friedman [3], D. Henry [5], A. Pazy [16], J. Smoller [18], and F. Rothe [19]).

Proposition 2.1. The system (1.1)-(1.3) admits a unique, classical solution $(u, v)$ on $\left[0, T_{\max }\left[\times \Omega\right.\right.$. If $T_{\max }<\infty$ then

$$
\lim _{t \nearrow T_{\max }}\left\{\|u(t, .)\|_{\infty}+\|v(t, .)\|_{\infty}\right\}=\infty
$$

where $T_{\max }$ denotes the eventual blowing-up time in $\mathbb{L}^{\infty}(\Omega)$.

We obtained in S. Kouachi[ 13], in particular for coupled reaction diffusion systems the following result

Proposition 2.2. Suppose that the functions $f(r, s)$ and $g(r, s)$ have polynomial growth and satisfy conditions (1.7). Then all positive solutions of (1.1)-(1.3) with initial data in $\mathbb{L}^{\infty}(\Omega)$ are global. tional

The main ingredient of the proof: We used the following polynomial func-

$$
t \longrightarrow L(t)=\int_{\Omega}\left[\sum_{i=0}^{p} C_{p}^{i} \theta_{i} u^{i} v^{p-i}\right] d x
$$

By differentiating $L$ with respect to $t$ and then by simple use of Green's formula we got

$$
L^{\prime}(t)=I+J
$$

where

$I=-p(p-1) \sum_{i=0}^{p-2} C_{p-2}^{i} \int_{\Omega} u^{i} v^{p-2-i}\left(a \theta_{i+2}|\nabla u|^{2}+(a+b) \theta_{i+1} \nabla u \nabla v+b \theta_{i}|\nabla v|^{2}\right) d x$ 
and

$$
J=\int_{\Omega}\left[p \sum_{i=0}^{p-1}\left(\theta_{i+1} f(u, v)+\theta_{i} g(u, v)\right) C_{p-1}^{i} u^{i} v^{p-1-i}\right] d x .
$$

Then we choose

$$
\frac{\theta_{i} \theta_{i+2}}{\theta_{i+1}^{2}} \geq \frac{(a+b)^{2}}{4 a b}, i=0,1, \ldots p-2,
$$

to get $I \leq 0$ and while using conditions (1.7) for $m=2$ to get

$$
J \leq C_{5} \int_{\Omega}\left[\sum_{i=0}^{p-1}(u+v+1) C_{p-1}^{i} u^{i} v^{p-1-i}\right] d x .
$$

Then, the functional $L$ satisfies the differential inequality

$$
L^{\prime}(t) \leq D_{1} L(t)+D_{2} L^{(p-1) / p}(t),
$$

where $D_{1}$ and $D_{2}$ are positive constants. While putting

$$
Z=L^{1 / p},
$$

one got

$$
p Z^{\prime} \leq D_{1} Z+D_{2} .
$$

The resolution of this linear differential inequality gives the uniform boundedness of the functional $L$ on the interval $\left[0, T_{\max }\right.$ [, what finished, by using the preliminary observations of this section, the proof.

By direct application of proposition 2.2, we obtained, in the case $h_{1}=h_{3}$ and $h_{2}=h_{4}$, the following result for problem (1.1)-(1.3):

Corollary 2.3. Suppose that

$$
\left\{\begin{array}{c}
l+q \leq 1 \text { or } r+s \leq 1, \\
\text { or } r+s>l+q>1 \text { and } l-r<s l-q r<s-q, \\
\text { or } l+q>r+s>1 \text { and } s-q<s l-q r<l-r .
\end{array}\right.
$$

Then, solutions of (1.1) with the boundary conditions (1.2) and the positive initial data (1.3) exist for all $t>0$; that is $T_{\max }=\infty$.

Recently, M. Pierre [17] generalized our results, in the case where

$$
h_{2} h_{3} \leq h_{1} h_{4}
$$

and proved global existence of solutions if

$$
\left\{\begin{array}{c}
s>q \text { and } s l-q r \leq s-q \text { or } s=q \text { and } l<r, \\
\text { or } l>r \text { and } s l-q r \leq l-r \text { or } l=r \text { and } s<q
\end{array}\right.
$$

and global weak solutions (solutions that are not in $\mathbb{L}^{\infty}(\Omega)$ but continue to live in $\left.\mathbb{L}^{1}(\Omega)\right)$ for all $l, q, r, s \geq 1$.

In this paper we present some generalizations of the above results and particularly the case

$$
\left\{\begin{array}{c}
s<q \text { and } l<r \\
\text { or } s>q \text { and } l>r
\end{array}\right.
$$

which remains an open problem. We prove global existence without condition (2.12) or (2.13) and solve the second case of (2.14). 


\section{Statement and proof of the main results}

The first result is the following

Proposition 3.1. 1. under conditions (2.11), the solutions of problem (1.1)(1.3) exists globally in time for all $h_{1}, h_{4}, h_{2}, h_{3}>0$.

2. under conditions (2.12) and for $h_{1}$ or $h_{4}$ sufficiently large or $h_{2}$ or $h_{3}$ sufficiently small, the solutions of problem (1.1)-(1.3) exists globally in time for all $l, q, r$, $s \geq 0$.

PROOF. We begin by the first case

(1) By differentiating $L$ given by (2.4) with respect to $t$, we get (2.5), where the integral $I$ given by (2.6) is negative under condition (2.8) on the sequence $\left\{\theta_{i}\right\}$. For the second integral $J$, we have

$$
J=p \sum_{i=0}^{p-1} \int_{\Omega}\left[\left(-\theta_{i+1} h_{1}+\theta_{i} h_{3}\right) u^{r} v^{s}+\left(\theta_{i+1} h_{2}-\theta_{i} h_{4}\right) u^{l} v^{q}\right] C_{p-1}^{i} u^{i} v^{p-1-i} d x .
$$

The case $r+s \leq 1$ is trivial while applying young inequality to the term $u^{r} v^{s}$ in the right hand side of the second equation of system (1.1) and choosing $\frac{\theta_{1}}{\theta_{0}}$ sufficiently small such that

$$
\theta_{i+1} h_{2}-\theta_{i} h_{4} \leq 0, i=0,1, \ldots p-1,
$$

for $p$ sufficiently large, then we get (2.9) which gives a differential inequality analogous to (2.10) and then the uniform boundedness of the functional $L$ on the interval $\left[0, T^{*}\right]$. We treat by the same way the case $l+q \leq 1$ while exchanging the roles of $r$ and $s$ with $l$ and $q$ and choosing $\frac{\theta_{1}}{\theta_{0}}$ sufficiently large such that

$$
-\theta_{i+1} h_{1}+\theta_{i} h_{3} \leq 0, i=0,1, \ldots p-1,
$$

for $p$ sufficiently large.

Suppose that the second line of (2.11) is satisfied. We'll prove that the functional $L$ given by (2.4) satisfies the differential inequality (2.10) and deduce its uniform boundedness on the interval $\left[0, T^{*}\right]$ :

Put

$$
\nu_{1}=\frac{r+s-1}{l+q-1} \text { and } \nu_{2}=\frac{\nu_{1}}{\nu_{1}-1}=\frac{r+s-1}{(r+s)-(l+q)},
$$

then

$$
\nu_{1}>1, \nu_{2} \text { and } \frac{1}{\nu_{1}}+\frac{1}{\nu_{2}}=1 .
$$

We can write

$$
l=l_{1}+l_{2} \text { and } q=q_{1}+q_{2},
$$

where

$$
\begin{gathered}
l_{1}=\frac{r}{\nu_{1}}=\frac{l+q-1}{r+s-1} r, l_{2}=\frac{(s l-q r)-(l-r)}{r+s-1} \\
q_{1}=\frac{s}{\nu_{1}}=\frac{l+q-1}{r+s-1} s \text { and } q_{2}=\frac{(s-q)-(s l-q r)}{r+s-1} .
\end{gathered}
$$


By choosing $\frac{\theta_{1}}{\theta_{0}}$ sufficiently large such that (3.3) is satisfied; for the terms for whom (3.2) is satisfied, we have

$\left(-\theta_{i+1} h_{1}+\theta_{i} h_{3}\right) u^{r} v^{s}+\left(\theta_{i+1} h_{2}-\theta_{i} h_{4}\right) u^{l} v^{q} \leq 0, i=0,1, \ldots p-1$.

For the remained terms, we apply Young inequality to get

$u^{l} v^{q} \leq\left(\frac{\theta_{i+1} h_{1}-\theta_{i} h_{3}}{\theta_{i+1} h_{2}-\theta_{i} h_{4}}\right)\left(u^{l_{1}} v^{q_{1}}\right)^{\nu_{1}}+C_{i}\left(u^{l_{2}} v^{q_{2}}\right)^{\nu_{2}}, i=0,1, \ldots p-1$,

where $C_{i}(i=0,1, \ldots p-1)$ are positive constants and

$$
\left(u^{l_{1}} v^{q_{1}}\right)^{\nu_{1}}=u^{r} v^{s} \text { and } \nu_{2}\left(l_{2}+q_{2}\right)=1 \text {. }
$$

Finally, while applying Young inequality another time to the second term of the right hand side of inequality (3.4), one from there deducts that

$$
\begin{gathered}
{\left[\left(-\theta_{i+1} h_{1}+\theta_{i} h_{3}\right) u^{r} v^{s}+\left(\theta_{i+1} h_{2}-\theta_{i} h_{4}\right) u^{l} v^{q}\right] \leq} \\
C_{i}^{\prime}(u+v), i=0,1, \ldots p-1
\end{gathered}
$$

where $C_{i}^{\prime}(i=0,1, \ldots p-1)$ are positive constants. From this last inequality we deduce the uniform boundedness of the functional $L$ on the interval $\left[0, T_{\max }[\right.$.

For the third line of $(2.11)$, following the same reasoning while taking

$$
\nu_{1}=\frac{l+q-1}{r+s-1} \text { and } \nu_{2}=\frac{\nu_{1}}{\nu_{1}-1}=\frac{l+q-1}{(l+q)-(r+s)}
$$

one prove that there exists positive constants $C_{i}^{\prime}(i=0,1, \ldots p-1)$ such that

$u^{r} v^{s} \leq\left(\frac{\theta_{i+1} h_{2}-\theta_{i} h_{4}}{\theta_{i+1} h_{1}-\theta_{i} h_{3}}\right)\left(u^{r_{1}} v^{s_{1}}\right)^{\nu_{1}}+C_{i}^{\prime \prime}\left(u^{r_{2}} v^{s_{2}}\right)^{\nu_{2}}, i=0,1, \ldots p-1$,

where $C_{i}^{\prime \prime}(i=0,1, \ldots p-1)$ are positive constants.

$$
r_{1}=\frac{l}{\nu_{1}}, r_{2}=r-r_{1}, s_{1}=\frac{q}{\nu_{1}} \text { and } s_{2}=s-s_{1} .
$$

From these we have

$$
\left(u^{r_{1}} v^{s_{1}}\right)^{\nu_{1}}=u^{l} v^{q} \text { and } \nu_{2}\left(r_{2}+s_{2}\right)=1 .
$$

Finally we deduce an analogous inequality to (3.5), which gives the uniform boundedness of the functional $L$ on the interval $\left[0, T_{\max }[\right.$.

(2) We'll prove that the functional $L$ given by (2.4) is decreasing: By differentiating it with respect to $t$, we get (2.5), where $I$ given by (2.6) is negative under condition $(2.8)$ on the sequence $\left\{\theta_{i}\right\}$. The integral $J$ is negative if we choose

$$
\frac{h_{3}}{h_{1}} \leq \frac{\theta_{i+1}}{\theta_{i}} \leq \frac{h_{4}}{h_{2}}, i=0,1, \ldots p-1 .
$$

Then, if $h_{1}$ or $h_{4}$ sufficiently large or $h_{2}$ or $h_{3}$ sufficiently small, the interval $\left[\frac{h_{3}}{h_{1}}, \frac{h_{4}}{h_{2}}\right]$ is sufficiently large to construct in it, for $p$ sufficiently large, the first $p$ elements of the sequence $\left\{\theta_{i}\right\}$. This implies the uniform boundedness of $\|f(u, v)\|_{p}$ and $\|g(u, v)\|_{p}$ on $\left[0, T^{*}[\right.$ and global existence becomes from the regularizing effect principle. That completes the proof. 
REMARK 3.2. The results of proposition 3.1, namely the second one, hold for more general nonlinearities

$$
f(u, v)=-f_{1}(u, v)+f_{2}(u, v), g(u, v)=g_{1}(u, v)-g_{2}(u, v),
$$

with $f_{i} \geq 0, g_{i} \geq 0$ satisfying

$$
\lim _{|u|+|v| \rightarrow+\infty} \frac{f_{1} g_{2}}{f_{2} g_{1}}=+\infty
$$

to get inequalities analogous to (3.6) for large integers $p$.

M. Pierre [17], proposed the following model

$$
\left\{\begin{array}{cc}
\frac{\partial u}{\partial t}-a \Delta u=\lambda u^{l} v^{q}-u^{r} v^{s} & \text { in } \mathbb{R}^{+} \times \Omega, \\
\frac{\partial v}{\partial t}-b \Delta v=-u^{l} v^{q}+u^{r} v^{s} & \text { in } \mathbb{R}^{+} \times \Omega,
\end{array}\right.
$$

where $\lambda \in[0,1]$. He proved global weak existence for all $l, q, r, s \geq 1$ and global existence only for $s l-q r<l-r$, but proposition 2.1 is applicable here by putting

$$
\lambda=h_{2} \text { and } h_{1}=h_{3}=h_{4}=1
$$

to deduce the following result

Corollary 3.3. For sufficiently small $\lambda$, the solutions of problem (1.1)" with boundary conditions (1.2) and positive uniformly bounded conditions (1.3) exist globally in time for all $l, q, r, s \geq 0$.

All the above results are not applicable to the case $l>r$ and $s>q$ (or $l<r$ and $s<q$ ). This case describes the chemical reaction diffusion model described by (1.1)'. Here we have

$$
h_{1} h_{4}=h_{2} h_{3}=(s-q)(l-r) \lambda_{1} \lambda_{2}
$$

and hypothesis of proposition 3.1 are not satisfied. Actually, global existence of solutions (even weak) is open. Namely, we have obtained the following result

Proposition 3.4. Suppose that $l<r$ and $s<q$ for some $l, q, r, s \geq 0$ and $h_{1} h_{4}=h_{2} h_{3}$, then all solutions of system (1.1) with boundary conditions (1.2) and positive uniformly bounded conditions (1.3) exist globally in time.

PRoOF. We can write the reaction as follows

$$
f(u, v)=u^{l} v^{q}-h u^{r} v^{s}, g(u, v)=k\left(-u^{l} v^{q}+h u^{r} v^{s}\right) .
$$

We have, for $p, p^{\prime}>1$ and $\gamma>0$

$$
\begin{aligned}
& \frac{d}{d t} \int_{\Omega}\left(u^{p}+\gamma v^{p^{\prime}}\right) d x \\
= & \int_{\Omega}\left(a p u^{p-1} \Delta u+b \gamma p^{\prime} v^{p-1} \Delta v\right) d x+\int_{\Omega}\left(p u^{p-1}-\gamma p^{\prime} k v^{p^{\prime}-1}\right)\left(u^{l} v^{q}-h u^{r} v^{s}\right) d x \\
= & I+J .
\end{aligned}
$$

By application of Green formula to the first integral and taking $u^{l} v^{s}$ as factor in the second, we get

$$
I=-\int_{\Omega}\left(a p(p-1) u^{p-2}|\nabla u|^{2}+b \gamma p^{\prime}\left(p^{\prime}-1\right) v^{p^{\prime}-2}|\nabla v|^{2}\right) d x
$$


and

$$
\begin{aligned}
J & =-\int_{\Omega} u^{l} v^{s}\left(p u^{p-1}-\gamma p^{\prime} k v^{p^{\prime}-1}\right)\left(h u^{r-l}-v^{q-s}\right) d x \\
& =-p h \int_{\Omega} u^{l} v^{s}\left(u^{p-1}-\frac{\gamma p^{\prime} k}{p} v^{p^{\prime}-1}\right)\left(u^{r-l}-h^{-1} v^{q-s}\right) d x
\end{aligned}
$$

Then $I \leq 0$. By choosing $p$ and $p^{\prime}$ satisfying

$$
\frac{p-1}{r-l}=\frac{p^{\prime}-1}{q-s}=\mu
$$

and putting

we get

$$
U=u^{r-l} \text { and } V=v^{q-s},
$$

$$
J=-p h \int_{\Omega} U^{\frac{l}{r-l}} V^{\frac{s}{q-s}}\left(U^{\mu}-\frac{\gamma p^{\prime} k}{p} V^{\mu}\right)\left(U-h^{-1} V\right) d x .
$$

By choosing $\gamma$ satisfying

$$
\frac{\gamma p^{\prime} k}{p}=h^{-\mu}
$$

we can deduce that

$$
U^{\mu}-\frac{\gamma p^{\prime} k}{p} V^{\mu}=\left(U^{\mu}-\left(h^{-1} V\right)^{\mu}\right)=\left(U-h^{-1} V\right) \sum_{i=0}^{\mu-1} U^{\mu-1-i}\left(h^{-1} V\right)^{i}
$$

and this gives $J \leq 0$.

REMARK 3.5. The large time behavior of this type of problems is standard and can be obtained by using compactness theorems to prove that

$$
\lim _{t \rightarrow+\infty} u(t, x)=u^{*} \text { and } \lim _{t \rightarrow+\infty} v(t, x)=v^{*},
$$

where $u^{*}$ and $v^{*}$ are positive constants satisfying $f\left(u^{*}, v^{*}\right)=g\left(u^{*}, v^{*}\right)=0$.

Acknowledgement The author would like to thank the anonymous referee for his precious suggestions, namely for his encouragement to enrich the paper with the generalization mentioned in remark 3.1 and the large time behavior of the global solutions described in remark 3.2 .

\section{References}

[1] S. Abdelmalek and S. Kouachi, Proof of Existence of Global Solutions for m-Components Reaction Diffusion Systems with Mixed Boundary Conditions via the Lyapunov Functional Method, J. Phys. A: Math. Theory. 40 (2007) 12335-12350.

[2] N. Alikakos, L ${ }^{p}$-Bounds of Solutions of Reaction-Diffusion Equations. Comm.. P. D. E. 4 (1979). 827-828.

[3] A. Friedman, Partial Differential Equations of Parabolic Type. Prentice Hall Englewood Chiffs. N. J. 1964.

[4] A. Haraux and A. Youkana, On a Result of K. Masuda Concerning Reaction-Diffusion Equations. Tôhoku. Math. J. 40 (1988), 159-163.

[5] D. Henry, Geometric Theory of Semi-linear Parabolic Equations. Lecture Notes in Mathematics 840, Springer-Verlag, New-York, 1984.

[6] S. L. Hollis, On the Question of Global Existence for Reaction-Diffusion Systems with Mixed Boundary Conditions. Quarterly of Applied Mathematics LI, number 2, June 1993, 241-250. 
[7] S. L. Hollis, R. H. Martin and M. Pierre, Global Existence and Boundedness in Reaction Diffusion Systems. SIAM. J. Math. Anal, Vol. 18, number 3, May 1987.

[8] M. Kirane and S. Kouachi, Asymptotic Behavior for a System Describing Epidemics with Migration and Spatial Spread of Infection. Dynamical Systems and Applications, Vol 12, (1993), 121-130.

[9] M. Kirane and S. Kouachi, A strongly nonlinear reaction diffusion model for a deterministic diffusive epidemic. Japan Journal of Industrial and Applied Mathematics, Volume 12, number 1, February 1995.

[10] M. Kirane and S. Kouachi, Global Solutions to a System of Strongly Coupled ReactionDiffusion Equations. Nonlinear Analysis Theory, Methods and Applications, Vol 126, (1996).

[11] S. Kouachi and A. Youkana, Global existence for a class of reaction-diffusion systems. Bulletin of the Polish Academy of Sciences, Vol. 49, Number 3, (2001).

[12] S. Kouachi, Existence of global solutions to reaction-diffusion systems via a Lyapunov functional, Electron. J. Di erential Equations, 68 (2001), 1-10.

[13] S. Kouachi, Existence of global solutions to reaction-diffusion systems with nonhomogeneous boundary conditions via a Lyapunov functional Electronic Journal of Differential Equations, Vol. 2002(2002), No. 88, pp. 1-13.

[14] K. Masuda, On the Global Existence and Asymptotic Behavior of Solutions of ReactionDiffusion Equations. Hokkaido. Math. J. 12 (1983), 360-370.

[15] J. Morgan, Global Existence for Semilinear Parabolic Systems, SIAM J. Math. Anal. 20, 1128-1144 (1989).

[16] A. Pazy, Semigroups of Linear Operators and Applications to Partial Differential Equations. Applied Math. Sciences 44, Springer-Verlag, New York (1983).

[17] M. Pierre, Global Existence in Reaction-Diffusion Systems with Control of Mass: a Survey.

[18] F. Rothe, Global Solutions of Reaction-Diffusion Systems, Lecture Notes in Math. 1072, Springer-Verlag, Berlin (1984).

[19] J. Smoller, Shock Waves and Reaction-Diffusion Equations, Springer-Verlag, New York (1983).

Department of Mathematics, College of Science, Qassim University,P.O.Box 6644, Al-Gassim, Buraydah 51452, Saudi Arabia.

E-mail address: kouachi@hotmail.com 